\title{
Thrombosis and Haemostasis 2021 Editors' Choice Papers
}

\author{
Christian Weber ${ }^{1,2,3}$ Anne Rigby ${ }^{1}$ Gregory Y. H. Lip ${ }^{4,5}$ \\ ${ }^{1}$ Institute for Cardiovascular Prevention (IPEK), LMU Munich, Munich, \\ Germany \\ ${ }^{2}$ German Centre for Cardiovascular Research (DZHK), partner site \\ Munich Heart Alliance, Munich, Germany \\ Address for correspondence Gregory Y. H. Lip, MD, Liverpool Centre \\ for Cardiovascular Science, University of Liverpool, William Henry \\ Duncan Building, 6 West Derby Street, Liverpool L7 8TX, United \\ Kingdom (e-mail: gregory.lip@liverpool.ac.uk).
}

${ }^{3}$ Department of Biochemistry, Cardiovascular Research Institute Maastricht (CARIM), Maastricht University Medical Centre, Maastricht, The Netherlands

${ }^{4}$ Liverpool Centre for Cardiovascular Science, Institute of Ageing and Chronic Disease, University of Liverpool, Liverpool, United Kingdom

${ }^{5}$ Aalborg Thrombosis Research Unit, Department of Clinical

Medicine, Aalborg University, Aalborg, Denmark

Thromb Haemost 2022;122:163-170.

This year's Editor's Choice highlights the 2021 manuscripts published in Thrombosis and Haemostasis and its open access companion journal $T H$ Open that found most resonance within our academic community. As in the precedent year, the 2021 COVID-19 pandemic situation has dictated the pace and direction of our research and clinical management efforts which, although not exclusively, is well reflected by this years' Editor's Choice contents. ${ }^{1-4}$

At the end of 2020, we published a Theme Issue dedicated to COVID-19 as a guide to better comprehend newly identified vascular and inflammatory mechanistic aspects of the disease, ${ }^{5}$ its hypercoagulation state, ${ }^{6}$ and clinical implications for vascular patients with COVID-19. ${ }^{7,8}$ To address the rapidly evolving situation and following a first consensus paper, ${ }^{9}$ we were pleased to publish an interesting discussion manuscript by the VAS investigators on the need for a more integrated and global strategy to COVID-19, providing a useful overview of public health approaches consideration and the next steps necessary to best manage the pandemic. ${ }^{10}$ While research efforts throughout 2020 focused on characterizing the coagulation abnormalities observed in COVID19 patients giving cues to the next clinical approaches to adopt, the results from such strategies mostly came out in the following year 2021.

\section{Understanding and Managing COVID-19}

\section{Anticoagulation for COVID-19}

Among the first published anticoagulation results in COVID19 , the cohort study from Billett et al found that patients with
Christian Weber, MD, Institute for Cardiovascular Prevention, LMU Munich, Pettenkoferstra × e 9, 80336 Munich, Germany (e-mail: chweber@med.Imu.de).

moderate or severe disease benefited from anticoagulation and that apixaban had similar efficacy to enoxaparin in decreasing mortality. ${ }^{11}$ The large real-life observational CORIST Collaboration study could confirm that heparin lowered in-hospital mortality, particularly in severely ill COVID-19 patients and in those with strong coagulation activation. $^{12}$

The first randomized controlled trial with antithrombotic sulodexide in COVID- 19 by Gonzalez-Ochoa et a ${ }^{13}$ suggested a benefit of sulodexide in COVID-19 early stage with less frequent hospitalization. Current societal and international recommendations of standard dose thromboprophylaxis in hospitalized patients with COVID-19 were confirmed by the large pooled analysis study from Patell et al. ${ }^{14}$ Similarly, the 90-day follow-up of the INSPIRATION Trial ${ }^{15}$ supported the routine use of standard dose over intermediate dose prophylactic anticoagulation in intensive care unit patients with COVID-19. Likewise, the multicenter retrospective study of venous thromboembolism (VTE) in COVID-19 patients by Cohen et al ${ }^{16}$ endorsed standard prophylacticdose anticoagulation in hospitalized patients with COVID-19 while individual clinical and laboratory parameters may be instrumental for tailoring thromboprophylaxis strategies in high-risk subgroups. Such high-risk hospitalized COVID-19 patients may indeed benefit from treatment-dose instead of prophylactic- or intermediate-dose low-molecular-weight heparin (LMWH) as demonstrated by the results from the recent multicenter HEP-COVID randomized trial. ${ }^{17,18}$ The multicenter observational GeroCovid study, presented convincing data on the role of direct oral anticoagulants (DOACs) received

December 2, 2021

accepted

December 2, 2021 (c) 2022. Thieme. All rights reserved. Georg Thieme Verlag KG,

Rüdigerstraße 14,

70469 Stuttgart, Germany
DOI https://doi.org/ 10.1055/s-0041-1741072. ISSN 0340-6245. 
in reducing the risk of death in COVID-19 older patients. ${ }^{19}$ While the beneficial anticoagulation effects of heparin on the thrombotic arm of COVID-19 is easily explicable, intriguing data from Mycroft-West et al suggested that heparin may also prevent severe acute respiratory syndrome coronavirus 2 (SARS-CoV-2) from infecting cells, ${ }^{20}$ opening options for repurposing heparin and its derivatives as antiviral agents.

Importantly, the precise identification of patients, who will best benefit from such prophylactic anticoagulation strategies, is key to their efficacy. Efforts in (re)defining risk biomarkers has therefore been the subject of several studies published last year. As mentioned in last year's Editorial, elevated D-dimer levels were associated with poor prognosis including severity and mortality with COVID-19 infection, ${ }^{21}$ making it a biomarker of choice for COVID-19 severity diagnosis. The small retrospective, one center study from Valerio et $\mathrm{al}^{22}$ further identified specific dynamics associated with D-dimer as well as C-reactive protein levels during COVID-19 which may refine monitoring disease activity. Sjöström et al also proposed a 7-day trend to assess the changing rate of D-dimer and platelet count for dose adjustment in relation to disease severity. ${ }^{23}$ Interestingly, Bauer et al pointed out a subtle nature of coagulation changes early in disease progression, highlighting the importance of monitoring coagulation parameters throughout disease progression. ${ }^{24}$ Since D-dimer has relatively low specificity and detects only late hemostatic stage, Chaudhary et al laid out the rationale for a study to detect whole blood viscoelastic analysis by thromboelastography or rotational thromboelastometry which may be useful to guide antithrombotic therapy in COVID-19 patients. $^{25}$ Several authors $^{26,27}$ also systematically analyzed the viscoelastic changes in the clotting system and acknowledged its use for predicting thromboembolic complications and to monitor the efficacy of anticoagulation and fibrinolytic treatment in COVID-19 patients. In a small pilot study, Hardy et al investigated daily monitoring of fibrin-related markers to better detect thrombotic events in COVID-19 patients, and suggested these could be used as further transient warning signals as D-dimers stay constantly elevated in critical patients. ${ }^{28}$ In a retrospective study of COVID-19 hospitalized patients, Sweeney et al demonstrated an association of low ADAMTS-13 (a disintegrin and metalloproteinase with a thrombospondin type 1 motif, member 13) activity on admission with increased risk of mortality, providing a further maker to detect patients who may benefit from more aggressive anticoagulation treatment. ${ }^{29}$ While clinical efforts to limit COVID-19-induced coagulopathy were and are imminently required in the actual situation, we also welcomed some less sought after strategies which may open new horizons for understanding and treating the hypercoagulation state of the disease. In this respect, Léopold et al shed light on the contribution of platelets to thromboembolic complications encountered in COVID-19 patients as they characterized the platelet phenotype and reactivity to collagen on SARS-CoV-2 infected hospitalized patients. ${ }^{30}$ The role of platelets in COVID-19 was also highlighted by Ghirardello et al in a flow-chamber system suggesting im- paired platelet activity and thrombus formation in the early phase of COVID-19. ${ }^{31}$ Such findings may open new avenues for antiplatelet drugs in COVID-19. Violi et al reported on a case series study of COVID-19 patients with hypoalbuminemia and intravenous administration of human albumin, ${ }^{32}$ thereby suggesting an innovative approach to dampen hypercoagulability worth considering. Hemoperfusion devices mimicking the endothelial glycocalyx may represent another option to treat SARS-CoV-2 as shown by a case report from Pape et al. ${ }^{33}$ Through electrostatic attraction, such systems are able to capture viruses, bacteria, and cytokines and may potentially reduce viral antigen and cytokine load in the setting of a cytokine storm.

While a great part of our scientific and clinical community has been fully engaged in identifying and refining anticoagulation management strategies for COVID-19, the virology and immunology scientists have been extremely fast in delivering the first efficient vaccines against SARS-CoV-2, creating a blow of hope throughout the world. As it appeared that some rare but severe cases of thrombotic events occurred after inoculation with adenoviral vector-based vaccines, it was crucial that such events should be better understood and treated by the thrombosis community. Although suspended or restricted in many countries, at least 1 billion doses of the adenoviral ChAdOx1 nCov-19 vaccine (AstraZeneca) are currently being released to low- and middle-income countries, making identification and treatment of vaccine-associated thrombosis crucial, as vaccination currently appears to be our sole pandemic exit strategy.

\section{Understanding and Treating Vaccine Complications}

In late February last year, rare but severe thrombotic events concomitant with thrombocytopenia, were reported in otherwise healthy individuals shortly after vaccination with the adenoviral vector-based vaccine ChAdOx $1 \mathrm{nCov}-19$ and later with another adenoviral vector-based vaccine (Ad26.COV2.S Janssen; Johnson \& Johnson). Risks versus benefits were carefully assessed in a timely consensus paper from experts in the field, ${ }^{34}$ who proposed a tracking algorithm for vaccinated patients based on 10-point guideline for safe decisionmaking. In this novel disorder, termed "vaccine-induced immune thrombotic thrombocytopenia" (VITT), vaccine components appear to form complexes with platelet factor 4 (PF4) on platelet surface triggering formation of highavidity anti-PF4 antibodies able to activate platelets in VITT patients ${ }^{35}$ strongly mimicking immune heparin-induced thrombocytopenia (HIT). In this context, the recently developed humanized monoclonal antibodies tools by Vayne et $\mathrm{al}^{36}$ to study the antibody response in autoimmune HIT may also help understanding VITT pathophysiology. While similar, HIT and VITT are however not identical ${ }^{37}$ and "rapid" PF4/heparin assays have been shown unsuitable to diagnose VITT in contrast to enzyme-linked immunosorbent assaybased PF4/heparin immunoassays. ${ }^{38}$ Because these immune complexes bind and activate platelets via $\mathrm{Fc}$ gamma receptor IIA, von Hundelshausen et $\mathrm{al}^{39}$ interestingly advocated for considering off-label use of Bruton tyrosine kinase (Btk) inhibitors (approved for B cell malignancies) in VITT, as 
they are expected to target Fc gamma RIIA-mediated activation of platelet aggregation. A proof-of-concept could be indeed provided in a subsequent study showing that Btk inhibitors inhibit platelet aggregation in whole blood induced by VITT serum from patients treated with or without intravenous immunoglobulins. ${ }^{40}$

In addition to thrombosis, other immunological reactions to SARS-CoV-2 vaccines have suggested that autoantibodies against the spike protein S1 of SARS-CoV-2 may be responsible for conditions such as immune thrombocytopenia, vasculitis, Schönlein-Henoch purpura, autoimmune hepatitis, and Guillain-Barré syndrome. ${ }^{41}$ Case reports such as that from Farley et al describing ${ }^{42}$ a case of acquired hemophilia after SARS-CoV-2 vaccination should therefore be considered carefully. Importantly, all cases reported recovered after adequate treatment, and should not hamper but rather help towards our vaccination efforts by providing transparent communication and defining appropriate treatment.

\section{Refining Anticoagulation Management}

Informing the 2020 European Society of Cardiology (ESC) guidelines on atrial fibrillation (AF), the proposal for characterization and evaluation by Potpara et $\mathrm{al}^{43}$ described the multidimensional aspects of AF requiring moving from classification toward a structured characterization addressing specific domains with treatment and prognostic implications, the 4S-AF scheme: Stroke risk; Symptom severity; Severity of AF burden; and Substrate. Our journal also published the Executive Summary of the 2021 Focused Update Consensus Guidelines of the Asia Pacific Heart Rhythm Society (APHRS) on Stroke Prevention in AF, with a state of the art discussion of the evidence and management recommendations for this common arrhythmia. ${ }^{44}$ The APHRS guidelines, in line with the ESC AF guidelines, recommends use of an integrated care or holistic management approach, based on the Atrial fibrillation Better Care (ABC) pathway ${ }^{45}$ to improve outcome in the Asian AF population. The components of the $\mathrm{ABC}$ pathway are as follows: "A": Avoid stroke with anticoagulation, that is, well-managed warfarin (time in therapeutic range [TTR] $>65-70 \%$ ) or non-vitamin K antagonist oral anticoagulant; "B": Better symptom management with patient-centered symptom-directed decisions for rate or rhythm control; and "C": Cardiovascular risk and comorbidity management as well as lifestyle changes. The APHRS guidelines noted evidence from a systematic review and meta-analysis of $>285,000$ patients from different regions of the world (including Asia), showing how adherence to the ABC pathway is associated with a markedly lower risk of all-cause death, cardiovascular death, stroke, and major bleeding, as well as hospitalizations. ${ }^{46}$ Of note the integrated care approach can be applied to other clinical scenarios (e.g., stroke, aortovascular disease) given the need to manage the "whole" patient, and not just one aspect of the patient. ${ }^{47,48}$ Before characterization and treatment of AF, it is important to detect the arrhythmia given the potential impact on clinical outcomes. ${ }^{49,50}$ Indeed, even asymptomatic $\mathrm{AF}$ is not benign, as shown in a nice study of the risk of ischemic stroke in asymptomatic AF patients incidentally detected in primary care. ${ }^{51,52}$ Kitsiou et at ${ }^{53}$ put forward the importance of continuous cardiac monitoring for detecting AF after a stroke, enabling clinicians to promptly switch to oral anticoagulation and prevent new strokes. Nelson et al addressed the existing literature on different anticoagulation strategies efficacy for critical care patients with AF, pointing out to our urgent need for randomized trials with standardized outcomes for such patients. ${ }^{54} \mathrm{Ma}-$ chine-learning approaches which account for risk factors' dynamic nature and multimorbidity may improve clinical stroke risk assessment over clinical scores, as demonstrated by a comparative study based on a large real-world data set. ${ }^{55}$ Jones et al investigated the economic aspects of service interventions and put into light the cost-effectiveness of anticoagulation clinics, in particular targeting patients at high-risk and/or with suboptimal treatment, ${ }^{56}$ a subject less often studied but nonetheless crucial in improving management praxis. While we require to acknowledge the dynamics of stroke risks and implement management flexibility accordingly, identifying specific groups of patients whose risks and benefits need to be weighted, also determines the success of anticoagulation strategies. Indeed, a balance between simplicity, practicality, and clinical application is needed. Biomarker-based scores can be nonspecific (predicting adverse events beyond stroke and bleeding), and more complex clinical scores are not necessarily the answer given that statistical significance is not the same as clinical significance. ${ }^{57,58}$

In our last Editor's Choice, we mentioned the difficulty in assessing risk-benefit tradeoff of antithrombotic therapies in East Asians patients who are more prone to bleeding. An interesting analysis by de Vries et al showed why event rates are higher in clinical practice than randomized trials of stroke prevention in $\mathrm{AF}^{59}$ Also, Pandey et al published a systematic review and meta-analysis comparing lower versus standard international normalized ratio (INR) targets in AF, showing evidence that low INR targets may be associated with lower bleeding but has more adverse outcomes. ${ }^{60,61}$ This work informed the 2021 APHRS guidelines on $\mathrm{AF}$, and highlights the importance of standard targets and good quality anticoagulation control, with high TTR. The consensus document from Kim et al on the safety and efficacy of different antithrombotic agents in Asians compared with Caucasian patients was welcomed by the clinical community as reflected by its high citation score. ${ }^{62}$ How AF patients with intermediate risk can benefit from anticoagulation is an important issue, which was addressed in a nationwide population-based study by Choi et $\mathrm{al}^{63}$ who observed benefits appear to have an age threshold. Also, some "real-world" evidence on the timing of starting oral anticoagulation, after an acute ischemic stroke with AF was provided by Chang et al, who showed the potential for more bleeding if anticoagulation was started early. ${ }^{64}$ Although risks associated with anticoagulation treatment in high body weight and obese patients is uncertain, the International Society of Thrombosis and Haemostasis guidance requires routinely checking DOAC concentrations in these 
patients which was challenged by two studies published last year ${ }^{65-67}$ that confirmed that almost all high body weight patients achieve expected drug levels with the use of apixaban, edoxaban, and rivaroxaban with no compromise in efficacy. Patients who suffered from major hemorrhage represent another group for whom the benefits and tradeoffs of anticoagulation remain uncertain. ${ }^{68}$ Although it seems natural to fear bleeding more than thrombosis in patients after major hemorrhage, Milling et al challenged this bias as they observed net benefit with restarting anticoagulation in a post hoc analysis on the DOAC reversal study ANNEXA-4, highlighting the need for randomized clinical trials in major bleeding scenarios. ${ }^{69}$ The well relayed consensus document from Douxfils et $\mathrm{al}^{70}$ also provided useful updated International Council for Standardization in Haematology guidance for laboratories regarding timing and modalities of monitoring DOACs, now including the newly Food and Drug Administration-approved DOAC betrixaban, and the specific DOAC reversal agent andexanet alfa. In our 2020 Editor's Choice we also highlighted reassuring evidence on the safety of DOACs in cancer patients, ${ }^{67,71-73}$ an issue again discussed by Falanga et al last year. ${ }^{74}$ The Swedish Patient register cohort study confirmed current AF guidelines on DOACs originally aimed for the general AF population. ${ }^{75}$ Supporting evidence also came from a study from Cohen et $\mathrm{al}^{76}$ among patients with VTE and active cancer prescribed apixaban, LMWH, or warfarin. In a post hoc analysis of the Caravaggio study, a large trial on the treatment of VTE in patients with cancer comparing apixaban with dalteparin, Ageno et al ${ }^{77}$ reported additional data on bleeding events which supports administering apixaban to an even larger population of cancer patients than previously recommended by guidelines, also including patients with gastrointestinal cancer, and should help clinicians translate the findings of the CARAVAGGIO study into clinical practice. Pregnancy and the postpartal phase are also associated with increasing VTE risk making it a leading cause of maternal mortality. We therefore very much welcomed the insights from the Global Anticoagulant Registry in the FIELD (GARFIELD)-VTE into the clinical characteristics, diagnostic strategies, treatment patterns, and outcomes of women with pregnancy-associated VTE. ${ }^{78}$ To refine bleeding risk for VTE patients under anticoagulant therapy, a valuable study compared the "classic" Registro Informatizado de Enfermedad TromboEmbólica (RIETE) score to the more recently developed VTE-BLEED score at different times after VTE diagnosis, with similar results for the prediction of early and late bleeds, and only small differences depending on the time since VTE diagnosis and the site of hemorrhage. ${ }^{79}$ As autoimmune hemolytic anemia is increasingly recognized as a strong risk factor for venous thrombosis but there are not yet any guidelines on thromboembolism prevention and management in this context, case report studies such as that of from Solari et al should prove very useful to the clinical community. ${ }^{80}$ Cirrhosis represents another disorder often associated with splanchnic vein thrombosis for which the meta-analysis from Valeriani et al reviewed useful clinical information on the value of anticoagulation therapy to lower the thrombotic burden without increasing overall bleeding risk. ${ }^{81}$ The efforts from Sokou et al ${ }^{82}$ to develop an easy-to-use risk score for critically ill neonates based on bed-side assessment of global coagulation and routinely available parameters was most welcome as these subjects have a high bleeding risk difficult to predict with conventional coagulation assays.

Following the ESC guidelines on acute coronary syndrome, the state-of-the-art review by Guedeney and Collet provided a comprehensive overview of current evidence on the pharmacological management of patients with acute coronary syndrome, particularly on the use of modern and more potent antiplatelet agents. ${ }^{83}$ The risk-benefit tradeoff of antithrombotic therapies in East Asian patients was informed by the pharmacodynamic randomized trial AMATCH which evaluated a prasugrel deescalation strategy in East Asian patients with acute coronary syndrome and showed a higher chance within the therapeutic window with reduced bleeding episodes. ${ }^{84}$

\section{Bleeding Risks and the Management of Bleeding Complications}

Hemophilia A patients whose lack of coagulation factor VIII (FVIII), put them at risk of prolonged bleeding are conventionally treated with FVIII infusion which is unfortunately limited by the development of neutralizing antibodies. The fourth generation rFVIII product simoctocog alfa (Nuwiq; Octapharma) replicating the native human FVIII protein without incorporation of potentially immunogenic elements of animal cell origin may represent a promising candidate to overcome this issue as reflected by the final data of the NuProtect study. ${ }^{85}$ The recently approved bispecific monoclonal antibody emicizumab represents another very attractive approach for treating hemophilia A patients bypassing FVIII activation. We highlighted last year the published promising clinical trials and preliminary reports with emicizumab $^{86}$ as well as its cost-effectiveness and budget. ${ }^{87} \mathrm{We}$ were pleased to publish new data from the Haven 1 study concerning pharmacokinetic and pharmacodynamic of emicizumab effects ${ }^{88}$ which should help the increasing number of clinicians using this new therapy. Severe bleeding is also one of the most common complications for cirrhosis patients with low fibrinogen for whom it is common practice to transfuse blood products such as cryoprecipitate. Budnick et al demonstrated the inefficacy of such treatment in improving survival or bleeding complications, seriously questioning routine management. ${ }^{89}$

\section{Identifying New Targets and Mechanisms}

Refining our understanding of platelet procoagulant physiology and pathophysiology has been an active subject of investigation in the context of COVID-1930,31,35 but also in a more general context as portrayed by further very welcomed investigations last year. Aliotta et a ${ }^{90}$ elegantly investigated ion fluxes in collagen and thrombin activated human platelets providing novel insights into the dichotomous role of the sodium-calcium exchanger in procoagulant platelet 
Fig. 1 Worldwide author distribution of original manuscripts submitted in 2021. Circle size is proportional to the total number of original manuscripts submitted in each country. Map created with Datawrapper.

formation. Ya et al have addressed the counteracting effects of protocatechuic acid on the apoptotic PI3K/Akt/GSK3 $\beta$ signaling pathways in human platelets, ${ }^{91}$ thereby highlighting a potentially important protective role in the progression of cardiovascular disease. The role of lipid rafts in cyclic adenosine monophosphate signaling homoeostasis in platelet was confirmed by Belleville-Rolland et $\mathrm{al}^{92}$ who further pointed out to the role of transporter multidrug resistance protein 4 in particular, and thus a new potential target for antithrombotic agents. The importance of the platelet-neutrophil feedback loop was evidence by a case-control study ${ }^{93}$ that put forward the importance of combining biomarkers of NETosis and platelet activation to predict the risk of major adverse events in a cohort of postmyocardial infarction patients. NETosis markers may also predict placenta-mediated complications in pregnancy as suggested by a pilot study, derived from the GrossPath study, demonstrating distinct circulating nucleosome-bound histones were increased in complicated pregnancy.${ }^{94}$ Further shedding light on the effect of neutrophil extracellular traps on coagulation, Locke and Longstaff ${ }^{95}$ published an interesting manuscript demonstrating the inhibitory effects of histones on fibrinolysis, suggesting new therapeutic targets for preventing thrombosis. Increasing evidence suggests that circulating micro-ribonucleic acids (miRNAs) could be used as diagnostic biomarkers and may even represent therapeutic targets for cardiovascular diseases. The study from Stojkovic et al assessing the relationship between different circulating miRNAs and monocyte-platelet aggregate formation in patients with acute coronary syndrome on dual antiplatelet therapy is an example of such new interest. ${ }^{96}$ Garcia et al identified an important role of a specific miRNA, miR-204$5 p$, on in vitro platelet production and function, ${ }^{97}$ suggesting genetic control of platelet production and function by miR204-5p which may thus be considered as a new biomarker for high platelet reactivity in the context of personalized antiplatelet therapy. ${ }^{98}$ The comprehensive overview of currently available techniques for studying miRNA function with a focus on platelet reactivity regulation by Garcia et al, ${ }^{99}$ also attracted much interest among our author community seeking to overcome the current methodological limitations in this field.

We are thereby delighted to publish an ever more diverse panel of scientific advances and clinical pictures reflecting the multifaceted aspects of Thrombosis and Haemostasis. This past year has brought its share of much needed investigations in understanding and treating the cardiovascular complications associated with COVID-19 in particular, but also in refining current clinical management in thrombosis in a more general context, opening new avenues for targeting and biomarker strategies as well as understanding underlying cardiovascular mechanisms. We are pleased that manuscripts are being increasingly submitted from all over the world: China now holds the record of submissions, followed by the United States, Italy, and Germany (- Fig. 1).

\section{Conflict of Interest}

G.Y.H.L. reports consultancy and speaker fees from Bayer, Bayer/Janssen, BMS/Pfizer, Biotronik, Medtronic, Boehringer Ingelheim, Microlife, Roche, and Daiichi-Sankyo outside the submitted Work. No fees received personally.

\section{References}

1 Lip GYH, Rigby A, Weber C. A Toast to the Last Decade and a Very Happy 2020 from Thrombosis and Haemostasis!. Thromb Haemost 2020;120(01):1-4

2 Lip GYH, Rigby A, Weber C. Looking Back on 2020, Looking Forward to 2021. Thromb Haemost 2021;121(01):1-3

3 Weber C, Rigby A, Lip GYH. Thrombosis and Haemostasis 2019 Editor's Choice Papers. Thromb Haemost 2020;120(01):184-190

4 Weber C, Rigby A, Lip GYH. Thrombosis and Haemostasis 2020 Editors' Choice Papers. Thromb Haemost 2021;121(01):109-114 
5 Gencer S, Lacy M, Atzler D, van der Vorst EPC, Döring Y, Weber C. Immunoinflammatory, thrombohaemostatic, and cardiovascular mechanisms in COVID-19. Thromb Haemost 2020;120(12):1629-1641

6 Katneni UK, Alexaki A, Hunt RC, et al. Coagulopathy and thrombosis as a result of severe COVID-19 infection: a microvascular focus. Thromb Haemost 2020;120(12):1668-1679

7 Schulman S, Hu Y, Konstantinides S. Venous thromboembolism in COVID-19. Thromb Haemost 2020;120(12):1642-1653

8 Waite AAC, Hamilton DO, Pizzi R, Ageno W, Welters ID. Hypercoagulopathy in severe COVID-19: implications for acute care. Thromb Haemost 2020;120(12):1654-1667

9 Gerotziafas GT, Catalano M, Colgan M-P, et al; Scientific Reviewer Committee. Guidance for the management of patients with vascular disease or cardiovascular risk factors and COVID-19: position paper from VAS-European Independent Foundation in Angiology/Vascular Medicine. Thromb Haemost 2020;120(12): 1597-1628

10 Gerotziafas GT, Catalano M, Theodorou Y, et al; Scientific Reviewer Committee. The COVID-19 pandemic and the need for an integrated and equitable approach: an international expert consensus paper. Thromb Haemost 2021;121(08):992-1007

11 Billett HH, Reyes-Gil M, Szymanski J, et al. Anticoagulation in COVID-19: effect of enoxaparin, heparin, and apixaban on mortality. Thromb Haemost 2020;120(12):1691-1699

12 Di Castelnuovo A, Costanzo S, Antinori A, et al. Heparin in COVID19 patients is associated with reduced in-hospital mortality: the multicenter Italian CORIST Study. Thromb Haemost 2021;121 (08):1054-1065

13 Gonzalez-Ochoa AJ, Raffetto JD, Hernández AG, et al. Sulodexide in the treatment of patients with early stages of COVID-19: a randomized controlled trial. Thromb Haemost 2021;121(07): 944-954

14 Patell R, Chiasakul T, Bauer E, Zwicker JI. Pharmacologic thromboprophylaxis and thrombosis in hospitalized patients with COVID-19: a pooled analysis. Thromb Haemost 2021;121(01): 76-85

15 Bikdeli B, Talasaz AH, Rashidi F, et al. Intermediate-dose versus standard-dose prophylactic anticoagulation in patients with COVID-19 admitted to the intensive care unit: 90-day results from the INSPIRATION randomized trial. Thromb Haemost 2021. Doi: $10.1055 / \mathrm{a}-1485-2372$

16 Cohen SL, Gianos E, Barish MA, et al; Northwell Health COVID-19 Research Consortium. Prevalence and predictors of venous thromboembolism or mortality in hospitalized COVID-19 patients. Thromb Haemost 2021;121(08):1043-1053

17 Goldin M, Giannis D, Diab W, et al. Treatment-dose LMWH versus prophylactic/intermediate dose heparins in high-risk COVID-19 inpatients: rationale and design of the HEP-COVID trial. Thromb Haemost 2021;121(12):1684-1695

18 Spyropoulos AC, Goldin M, Giannis D, et al. Efficacy and safety of therapeutic-dose heparin vs standard prophylactic or intermediate-dose heparins for thromboprophylaxis in high-risk hospitalized patients with COVID-19: the HEP-COVID randomized clinical trial. JAMA Intern Med 2021;181(12):1612-1620

19 Fumagalli S, Trevisan C, Del Signore S, et al. COVID-19 and atrial fibrillation in older patients: does oral anticoagulant therapy provide a survival benefit?-an insight from the GeroCovid Registry Thromb Haemost 2021. Doi: 10.1055/a-1503-3875

20 Mycroft-West CJ, Su D, Pagani I, et al. Heparin inhibits cellular invasion by SARS-CoV-2: structural dependence of the interaction of the Spike S1 receptor-binding domain with heparin. Thromb Haemost 2020;120(12):1700-1715

21 Lippi G, Favaloro EJ. D-dimer is associated with severity of coronavirus disease 2019: a pooled analysis. Thromb Haemost 2020;120(05):876-878

22 Valerio L, Ferrazzi P, Sacco C, et al; Humanitas COVID-19 Task Force. Course of D-dimer and C-reactive protein levels in survivors and nonsurvivors with COVID-19 pneumonia: a retrospec- tive analysis of 577 patients. Thromb Haemost 2021;121(01): 98-101

23 Sjöström A, Wersäll JD, Warnqvist A, et al. Platelet count rose while D-dimer levels dropped as deaths and thrombosis declined -an observational study on anticoagulation shift in COVID-19. Thromb Haemost 2021;121(12):1610-1621

24 Bauer W, Galtung N, Neuwinger N, et al. A matter of caution: coagulation parameters in COVID-19 do not differ from patients with ruled-out SARS-CoV-2 infection in the emergency department. TH Open 2021;5(01):e43-e55

25 Chaudhary R, Kreutz RP, Bliden KP, Tantry US, Gurbel PA. Personalizing antithrombotic therapy in COVID-19: role of thromboelastography and thromboelastometry. Thromb Haemost 2020; 120(11):1594-1596

26 Hammer S, Häberle H, Schlensak C, et al. Severe SARS-CoV-2 infection inhibits fibrinolysis leading to changes in viscoelastic properties of blood clot: a descriptive study of fibrinolysis in COVID-19. Thromb Haemost 2021;121(11):1417-1426

27 Słomka A, Kowalewski M, Żekanowska E. Hemostasis in coronavirus disease 2019-lesson from viscoelastic methods: a systematic review. Thromb Haemost 2021;121(09):1181-1192

28 Hardy M, Michaux I, Dive A, Lecompte T, Mullier F. Could daily monitoring of fibrin related markers help suspect a thrombotic event in COVID-19 patients? A prospective pilot study. TH Open 2021;5(02):e152-e154

29 Sweeney JM, Barouqa M, Krause GJ, Gonzalez-Lugo JD, Rahman S, Gil MR. Low ADAMTS13 activity correlates with increased mortality in COVID-19 patients. TH Open 2021;5(01):e89-e103

30 Léopold V, Pereverzeva L, Schuurman AR, et al. Platelets are hyperactivated but show reduced glycoprotein VI reactivity in COVID-19 patients. Thromb Haemost 2021;121(09):1258-1262

31 Ghirardello S, Lecchi A, Artoni A, et al. Assessment of platelet thrombus formation under flow conditions in adult patients with COVID-19: an observational study. Thromb Haemost 2021;121 (08):1087-1096

32 Violi F, Ceccarelli G, Loffredo L, et al. Albumin supplementation dampens hypercoagulability in COVID-19: a preliminary report. Thromb Haemost 2021;121(01):102-105

33 Pape A, Kielstein JT, Krüger T, Fühner T, Brunkhorst R. Treatment of a critically ill COVID-19 patient with the Seraph 100 Microbind Affinity Filter. TH Open 2021;5(02):e134-e138

34 Elalamy I, Gerotziafas G, Alamowitch S, et al; Scientific Reviewer Committee. SARS-CoV-2 vaccine and thrombosis: an expert consensus on vaccine-induced immune thrombotic thrombocytopenia. Thromb Haemost 2021;121(08):982-991

35 Greinacher A, Thiele T, Warkentin TE, Weisser K, Kyrle PA, Eichinger S. Thrombotic thrombocytopenia after ChAdOx1 nCov-19 vaccination. N Engl J Med 2021;384(22):2092-2101

36 Vayne C, Nguyen TH, Rollin J, et al. Characterization of new monoclonal PF4-specific antibodies as useful tools for studies on typical and autoimmune heparin-induced thrombocytopenia. Thromb Haemost 2021;121(03):322-331

37 Warkentin TE. Heparin-induced thrombocytopenia and vaccineinduced immune thrombotic thrombocytopenia antibodies: fraternal-not identical-twins. Thromb Haemost 2021;121(12): 1558-1561

38 Sachs UJ, Cooper N, Czwalinna A, et al. PF4-dependent immunoassays in patients with vaccine-induced immune thrombotic thrombocytopenia: results of an interlaboratory comparison. Thromb Haemost 2021;121(12):1622-1627

39 von Hundelshausen P, Lorenz R, Siess W, Weber C. Vaccineinduced immune thrombotic thrombocytopenia (VITT): targeting pathomechanisms with Bruton tyrosine kinase inhibitors. Thromb Haemost 2021;121(11):1395-1399

40 Weber C, von Hundelshausen P, Siess W. VITT after ChAdOx1 nCoV-19 vaccination. N Engl J Med 2021;385:2202-2205

41 Harenberg J, Marchetti M, Falanga A. Acquired autoimmune hemophilia following SARS-CoV-2 vaccines: dual-drug effects 
on blood coagulation and the Scylla and Charybdis phenomenon. Thromb Haemost 2021;121(12):1555-1557

42 Farley S, Ousley R, Van Wagoner N, Bril F. Autoimmunity after coronavirus disease 2019 (COVID-19) vaccine: a case of acquired hemophilia A. Thromb Haemost 2021;121(12):1674-1676

43 Potpara TS, Lip GYH, Blomstrom-Lundqvist C, et al. The 4S-AF scheme (Stroke Risk; Symptoms; Severity of Burden; Substrate): a novel approach to in-depth characterization (rather than classification) of atrial fibrillation. Thromb Haemost 2021;121(03): 270-278

44 Chao TF, Joung B, Takahashi Y, et al. 2021 Focused Update Consensus Guidelines of the Asia Pacific Heart Rhythm Society on Stroke Prevention in Atrial Fibrillation: Executive Summary. Thromb Haemost 2021;123(01):20-47

45 Lip GYH. The ABC pathway: an integrated approach to improve AF management. Nat Rev Cardiol 2017;14(11):627-628

46 Romiti GF, Pastori D, Rivera-Caravaca JM, et al. Adherence to the 'Atrial Fibrillation Better Care' pathway in patients with atrial fibrillation: impact on clinical outcomes-a systematic review and meta-analysis of 285,000 patients. Thromb Haemost 2021. Doi: 10.1055/a-1515-9630

47 Lip GYH, Ntaios G. "Novel Clinical Concepts in Thrombosis": integrated care for stroke management-easy as ABC. Thromb Haemost 2021. Doi: 10.1055/a-1632-1777

48 Field M, Kuduvalli M, Torella F, McKay V, Khalatbari A, Lip GYH. Integrated care systems and the aortovascular hub. Thromb Haemost 2021. Doi: 10.1055/a-1591-8033

49 Sun W, Freedman B, Martinez C, Wallenhorst C, Yan BP. Atrial fibrillation detected by single time-point handheld electrocardiogram screening and the risk of ischemic stroke. Thromb Haemost 2021. Doi: $10.1055 / \mathrm{a}-1588-8867$

50 Proietti M, Boriani G. Screening for atrial fibrillation in relation to stroke and mortality risk. Thromb Haemost 2021. Doi: 10.1055/a1562-0747

51 Wallenhorst C, Martinez C, Freedman B. Risk of ischemic stroke in asymptomatic atrial fibrillation incidentally detected in primary care compared with other clinical presentations. Thromb Haemost 2021. Doi: 10.1055/a-1541-3885

52 Wachter R, Freedman B. Subclinical atrial fibrillation and the risk of recurrent ischemic stroke. Thromb Haemost 2021;121(06): 697-699

53 Kitsiou A, Rogalewski A, Kalyani M, et al. Atrial fibrillation in patients with embolic stroke of undetermined source during 3 years of prolonged monitoring with an implantable loop recorder. Thromb Haemost 2021;121(06):826-833

54 Nelson AJ, Johnston BW, Waite AAC, Lemma G, Welters ID. A systematic review of anticoagulation strategies for patients with atrial fibrillation in critical care. Thromb Haemost 2021;121(12): 1599-1609

55 Lip GYH, Genaidy A, Tran G, Marroquin P, Estes C, Sloop S. Improving stroke risk prediction in the general population: a comparative assessment of common clinical rules, a new multimorbid index, and machine-learning-based algorithms. Thromb Haemost 2021. Doi: 10.1055/a-1467-2993

56 Jones NR, Crawford W, Yang Y, Hobbs FDR, Taylor CJ, Petrou S. A systematic review of economic aspects of service interventions to increase anticoagulation use in atrial fibrillation. Thromb Haemost 2021. Doi: 10.1055/a-1515-9428

57 Camelo-Castillo A, Rivera-Caravaca JM, Marín F, Vicente V, Lip $\mathrm{GYH}$, Roldán V. Predicting adverse events beyond stroke and bleeding with the ABC-stroke and ABC-bleeding scores in patients with atrial fibrillation: the Murcia AF Project. Thromb Haemost 2020;120(08):1200-1207

58 Proietti M, Rivera-Caravaca JM, Esteve-Pastor MA, Marín F, Lip GYH. Stroke and thromboembolism in warfarin-treated patients with atrial fibrillation: comparing the CHA2DS2-VASc and GARFIELD-AF risk scores. Thromb Haemost 2021;121(08):1107-1114
59 de Vries TAC, Hirsh J, Xu K, et al. Apixaban for stroke prevention in atrial fibrillation: why are event rates higher in clinical practice than in randomized trials?-a systematic review Thromb Haemost 2020;120(09):1323-1329

60 Chao T-F, Guo Y. Should we adopt a standard international normalized ratio range of 2.0 to 3.0 for Asian patients with atrial fibrillation? An appeal for evidence-based management, not eminence-based recommendations. Thromb Haemost 2020;120 (03):366-368

61 Pandey AK, Xu K, Zhang L, et al. Lower versus standard INR targets in atrial fibrillation: a systematic review and meta-analysis of randomized controlled trials. Thromb Haemost 2020;120(03): 484-494

62 Kim HK, Tantry US, Smith SC Jr, et al. The East Asian paradox: an updated position statement on the challenges to the current antithrombotic strategy in patients with cardiovascular disease. Thromb Haemost 2021;121(04):422-432

63 Choi SY, Kim MH, Lee KM, et al. Age-dependent anticoagulant therapy for atrial fibrillation patients with intermediate risk of ischemic stroke: a nationwide population-based study. Thromb Haemost 2021;121(09):1151-1160

64 Chang PY, Wang W, Wu WL, et al. Oral anticoagulation timing in patients with acute ischaemic stroke and atrial fibrillation. Thromb Haemost 2021. Doi: 10.1055/a-1669-4987

65 Pandey AK, Eikelboom JW. Direct oral anticoagulant dosing in extremes of body weight: time to revisit the guidelines? Thromb Haemost 2021;121(02):118-120

66 Martin AC, Thomas W, Mahir Z, et al. Direct oral anticoagulant concentrations in obese and high body weight patients: a cohort study. Thromb Haemost 2021;121(02):224-233

67 Boriani G, Ruff CT, Kuder JF, et al. Edoxaban versus warfarin in patients with atrial fibrillation at the extremes of body weight: an analysis from the ENGAGE AF-TIMI 48 trial. Thromb Haemost 2021;121(02):140-149

68 Edlmann E, McMahon C. Balancing risks and benefits when recommencing oral anticoagulants after major bleeding. Thromb Haemost 2021;121(08):979-981

69 Milling TJ Jr, King B, Yue P, et al; ANNEXA-4 Investigators. Restart of anticoagulant therapy and risk of thrombosis, rebleeding, and death after factor Xa inhibitor reversal in major bleeding patients. Thromb Haemost 2021;121(08):1097-1106

70 Douxfils J, Adcock DM, Bates SM, et al. 2021 update of the International Council for Standardization in Haematology recommendations for laboratory measurement of direct oral anticoagulants. Thromb Haemost 2021;121(08):1008-1020

71 Giustozzi M, Agnelli G, Del Toro-Cervera J, et al. Direct oral anticoagulants for the treatment of acute venous thromboembolism associated with cancer: a systematic review and metaanalysis. Thromb Haemost 2020;120(07):1128-1136

72 Cavallari I, Verolino G, Romano S, Patti G. Efficacy and safety of nonvitamin $\mathrm{K}$ oral anticoagulants in patients with atrial fibrillation and cancer: a study-level meta-analysis. Thromb Haemost 2020;120(02):314-321

73 Abrahami D, Renoux C, Yin H, Fournier JP, Azoulay L. The association between oral anticoagulants and cancer incidence among individuals with nonvalvular atrial fibrillation. Thromb Haemost 2020;120(10):1384-1394

74 Falanga A, Gal GL, Carrier M, et al. Management of cancerassociated thrombosis: unmet needs and future perspectives. TH Open 2021;5(03):e376-e386

75 Atterman A, Friberg L, Asplund K, Engdahl J. Atrial fibrillation, oral anticoagulants, and concomitant active cancer: benefits and risks. TH Open 2021;5(02):e176-e182

76 Cohen A, Keshishian A, Lee T, et al. Effectiveness and safety of apixaban, low-molecular-weight heparin, and warfarin among venous thromboembolism patients with active cancer: a U.S. claims data analysis. Thromb Haemost 2021;121(03):383-395 
77 Ageno W, Vedovati MC, Cohen A, et al. Bleeding with apixaban and dalteparin in patients with cancer-associated venous thromboembolism: results from the Caravaggio study. Thromb Haemost 2021;121(05):616-624

78 Jerjes-Sánchez C, Rodriguez D, Farjat AE, et al; GARFIELD-VTE investigators. Pregnancy-associated venous thromboembolism: insights from GARFIELD-VTE. TH Open 2021;5(01):e24-e34

79 Lecumberri R, Jiménez L, Ruiz-Artacho P, et al; RIETE investigators. Prediction of major bleeding in anticoagulated patients for venous thromboembolism: comparison of the RIETE and the VTEBLEED scores. TH Open 2021;5(03):e319-e328

80 Solari D, Alberio L, Ribi C, Grandoni F, Stalder G. Autoimmune hemolytic anemia and pulmonary embolism: an association to consider. TH Open 2021;5(01):e8-e13

81 Valeriani E, Di Nisio M, Riva N, et al. Anticoagulant treatment for splanchnic vein thrombosis in liver cirrhosis: a systematic review and meta-analysis. Thromb Haemost 2021;121(07):867-876

82 Sokou R, Piovani D, Konstantinidi A, et al. A risk score for predicting the incidence of hemorrhage in critically ill neonates: development and validation study. Thromb Haemost 2021;121 (02):131-139

83 Guedeney P, Collet J-P. Antithrombotic therapy in acute coronary syndromes: current evidence and ongoing issues regarding early and late management. Thromb Haemost 2021;121(07):854-866

84 Jeong Y-H, Oh J-H, Yoon H-J, et al. Pharmacodynamic profile and prevalence of bleeding episode in East Asian patients with acute coronary syndromes treated with prasugrel standard-dose versus de-escalation strategy: a randomized A-MATCH trial. Thromb Haemost 2021;121(10):1376-1386

85 Liesner RJ, Abraham A, Altisent C, et al. Simoctocog alfa (Nuwiq) in previously untreated patients with severe haemophilia A: final results of the NuProtect Study. Thromb Haemost 2021;121(11): $1400-1408$

86 Gelbenegger G, Schoergenhofer C, Knoebl P, Jilma B. Bridging the missing link with emicizumab: a bispecific antibody for treatment of hemophilia A. Thromb Haemost 2020;120(10): 1357-1370

87 Cortesi PA, Castaman G, Trifirò G, et al. Cost-effectiveness and budget impact of emicizumab prophylaxis in haemophilia $\mathrm{A}$ patients with inhibitors. Thromb Haemost 2020;120(02): $216-228$
88 Schmitt C, Adamkewicz JI, Xu J, et al. Pharmacokinetics and pharmacodynamics of emicizumab in persons with hemophilia A with factor VIII inhibitors: HAVEN 1 Study. Thromb Haemost 2021;121(03):351-360

89 Budnick IM, Davis JPE, Sundararaghavan A, et al. Transfusion with cryoprecipitate for very low fibrinogen levels does not affect bleeding or survival in critically ill cirrhosis patients. Thromb Haemost 2021;121(10):1317-1325

90 Aliotta A, Bertaggia Calderara D, Zermatten MG, Alberio L. Sodium-calcium exchanger reverse mode sustains dichotomous ion fluxes required for procoagulant COAT platelet formation. Thromb Haemost 2021;121(03):309-321

91 Ya F, Li K, Chen H, et al. Protocatechuic acid protects platelets from apoptosis via inhibiting oxidative stress-mediated PI3K/Akt/ GSK3 $\beta$ signaling. Thromb Haemost 2021;121(07):931-943

92 Belleville-Rolland T, Leuci A, Mansour A, et al. Role of membrane lipid rafts in MRP4 (ABCC4) dependent regulation of the cAMP pathway in blood platelets. Thromb Haemost 2021;121(12): 1628-1636

93 Hally KE, Parker OM, Brunton-O'Sullivan MM, Harding SA, Larsen PD. Linking neutrophil extracellular traps and platelet activation: a composite biomarker score for predicting outcomes after acute myocardial infarction. Thromb Haemost 2021;121(12):1637-1649

94 Bouvier S, Fortier M, Vincent L, et al. NETosis markers in pregnancy: effects differ according to histone subtypes. Thromb Haemost 2021;121(07):877-890

95 Locke M, Longstaff C. Extracellular histones inhibit fibrinolysis through noncovalent and covalent interactions with fibrin. Thromb Haemost 2021;121(04):464-476

96 Stojkovic S, Wadowski PP, Haider P, et al. Circulating microRNAs and monocyte-platelet aggregate formation in acute coronary syndrome. Thromb Haemost 2021;121(07):913-922

97 Garcia A, Dunoyer-Geindre S, Nolli S, Strassel C, Reny JL, Fontana P. miR-204-5p and platelet function regulation: insight into a mechanism mediated by CDC42 and GPIIbIIIa. Thromb Haemost 2021;121(09):1206-1219

98 Moon MJ, Peter K. MicroRNAs as biomarkers for personalized antiplatelet therapy. Thromb Haemost 2021;121(09):1121

99 Garcia A, Dunoyer-Geindre S, Fish RJ, Neerman-Arbez M, Reny JL, Fontana P. Methods to investigate miRNA function: focus on platelet reactivity. Thromb Haemost 2021;121(04):409-421 